\title{
Radiotherapy in benign orbital disease. I: Complicated ocular angiomas
}

\author{
P N PLOWMAN AND A N HARNETT \\ From the Department of Radiotherapy, St Bartholomew's Hospital, London EC1A 7BE
}

\begin{abstract}
SUMMARY Radiotherapy has been successful in the management of complicated ocular and orbital angiomas, but late morbidity (particularly radiation induced cataract) has been a problem. With modern radiotherapy techniques it is possible to minimise the morbidity to other tissues. By means of a recently developed method of lens-sparing ocular radiotherapy (initially adopted for retinoblastoma therapy) two cases of diffuse ocular haemangioma complicated by retinal detachment have been treated, with evidence of regression.
\end{abstract}

It has been well established that the majority of haemangiomas of childhood are harmless and require no treatment and that the majority of capillary haemangiomas will spontaneously regress. ${ }^{12}$ Although radiotherapy has historically been used to induce regression of paediatric haemangiomas, ${ }^{13}$ this treatment is now considered to be contraindicated in most cases. Furthermore, steroid therapy has been shown to induce regression of such haemangiomas, ${ }^{4}$ including orbital haemangiomas,,-5 and when therapy is indicated this form of treatment is preferred to radiation therapy, though its success rate has not been high in all workers' experiences. ${ }^{910}$

The commonest site for orbital haemangiomas in childhood is in the anterior orbit and upper lid. ${ }^{10}$ In the large New York series the high incidence of tumour-associated, late visual sequelae (namely, amblyopia, anisometria, strabismus, optic nerve atrophy) prompted the authors to advise early treatment with both steroids and radiotherapy for large lesions, though the data from treated cases still reflected a high incidence of tumour-associated sequelae."1"

The radiotherapeutic management of lid haemangiomas is relatively straightforward, as a lead eye shield, placed behind the lids and against the eye, protects the eye itself from the superficial $x$-rays which are used to treat the lid. This is an established technique that requires little further comment.

However, a number of complicated ocular angiomas remain (both haemangiomas and lymph-

Correspondence to Dr P N Plowman. angiomas), situated behind the lids, around the globe, within the eye itself, or posteriorly within the orbit, for which there has correctly been great concern for late morbidity associated with radiotherapy. Two such cases presenting recently to the joint ocular oncology unit of St Bartholomew's and Moorfields hospitals are presented here to illustrate a method of treatment designed to minimise morbidity associated with the treatment.

\section{Case reports}

CASE 1

This 12-year-old girl was born with a 'port wine' stain over her right upper lid associated with vascular conjunctival and episcleral injection of that eye. However, the child's eyesight had been checked by an optician at age 7 and was said to have been good in both eyes. The child presented to an ophthalmologist at 11 years because of decreased vision in the right eye. On examination there was an upper right facial port wine stain involving the upper lid, with markedly dilated episcleral and conjunctival vessels. The right corneal diameter was greater than the left. The intraocular pressure was raised in the right eye. Retinoscopy showed +7 dioptres of hypermetropia in the right eye, and on funduscopy showed a serous detachment of the posterior retina including the macula and pericapillary area, with a probable underlying haemangioma. The left fundus was normal. Visual acuity was reduced to counting fingers on the right.

The diagnosis was that of a variant of the Sturge- 


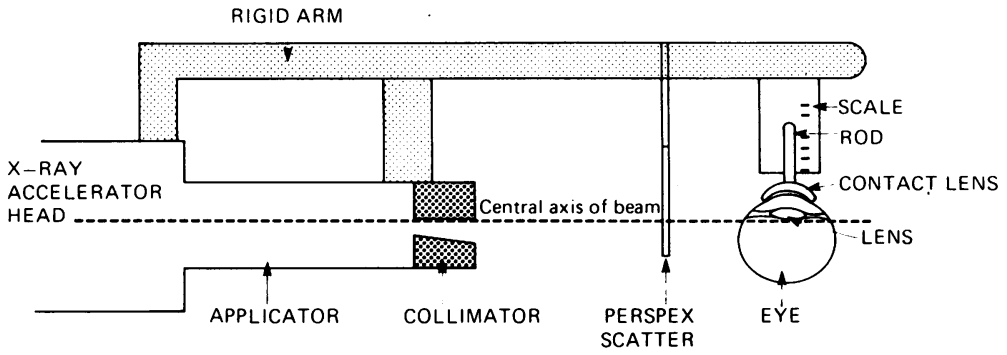

Fig. 1A Schematic drawing of lens-sparing ocular radiotherapy technique. The non-divergent, penumbra-trimmed anterior beam edge is positioned at the back of the lens (Harnett et al. ${ }^{12}$ ).

Weber syndrome, including a choroidal haemangioma and serous retinal detachment.

Treatment began with external beam radiotherapy. The technique used was that developed for retinoblastoma therapy - that is, a penumbra-free, non-divergent anterior beam edge from a linear accelerator source was positioned with high accuracy, via a contact lens reference point on the cornea, behind the lens of the eye to provide a lenssparing ocular portal (Figs. 1A, B). A tumour dose of 1200 cGy was delivered in eight fractions over nine days (6 MV photons).

Nine months later the retina was flat, the haemangioma smaller, and the intraocular pressure reduced, but the visual acuity remained at counting fingers. The fluorescein angiograms demonstrate the improvement before and after radiotherapy (Figs. 2A, B).

\section{CASE 2}

This girl was initially examined ophthalmologically at the age of 1 year, when she had a vascular lesion on the right cheek. She was then noted to have abnormal pigmentation of the iris inferiorly and extensive pigmentation in the right fundus inferiorly and temporally. There was no evidence of any angiomatous lesion in the fundus. At age 4 years a

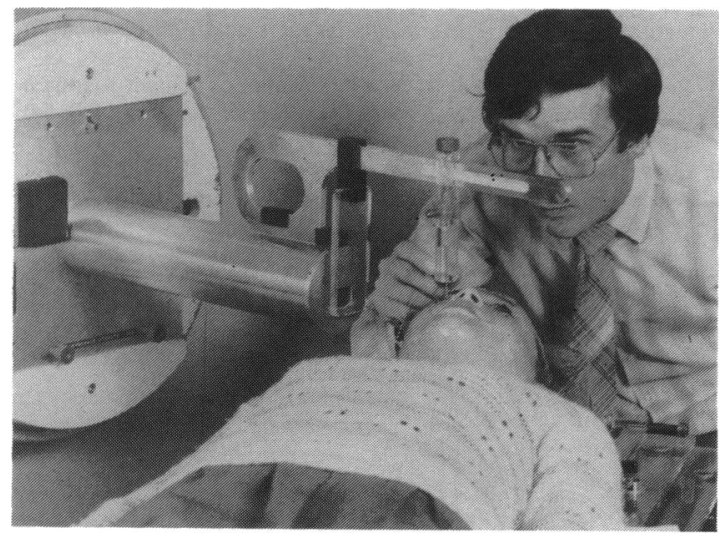

Fig. 1B Patient (case 1) being positioned for a treatment. considerable number of enlarged vessels were noted in the iris, and the fundus had angiomatous vessels and an area of pigmentation and choroidal atrophy. Visual acuities were 6/24 right eye, $6 / 4$ left eye. The visual acuity in the right eye had deteriorated to $6 / 60$ at the child's presentation to this centre at age $61 / 2$ years, and at this time there was an obvious and very large choroidal haemangioma in the inferior midperiphery with associated serous retinal detachment. By ultrasound examination the haemangioma was found to have a base diameter of $17 \mathrm{~mm}$ and it was 6 mm thick.

By means of the lens-sparing external beam, ocular radiotherapy technique (as for case 1) a tumour dose of $1200 \mathrm{cGy}$ was delivered in eight fractions over 12 days (6 MV $x$-rays). Six months later the retina was flat and the tumour was smaller. The visual acuity had improved to $6 / 36$.

\section{Discussion}

Although radiotherapy has been used to treat orbital angiomas for many years, the indications for such treatment have in recent years been confined to large and complicated cases. While discrete haemangiomas causing retinal detachment have previously been treated by laser photocoagulation or cobalt plaque therapy, the diffuse ocular haemangiomas presented in the two cases here could not be so treated.

When modern radiotherapeutic techniques are used, their sophistication allows treatment to occur with only a small risk of late treatment-related morbidity. This last fact bears directly on the timing of future recommendations for radiotherapy, particularly when the incidence of the late visual complications of angioma is high." Early radiotherapy for intraocular angiomas would not previously have been contemplated by ophthalmologists mindful of the literature on morbidity related to treatment."

In the cases reported here a modern method of lens-sparing ocular radiotherapy, developed for retinoblastoma therapy, ${ }^{12}$ was employed to treat ocular haemangiomas complicated by serous retinal detachments and visual deterioration. In both cases 


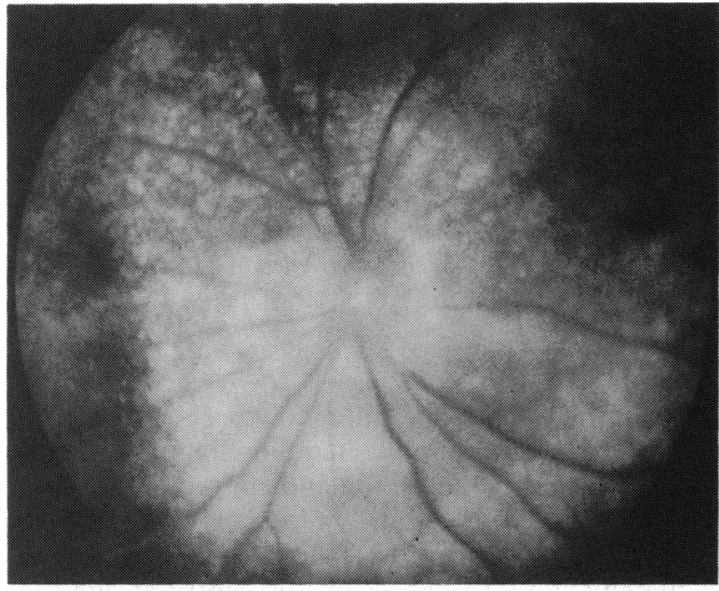

Fig. 2 Fluorescein angiogram photographs (case 1). Both figs. $2 A$ and $2 B$ represent the recirculation phase at 10 minutes after injection. Fig. $2 A$ was taken prior to radiotherapy and shows slow clearing of the dye.

the therapy was effective in causing regression of the angioma and reversal of the retinal detachment; return of vision was limited by the pretreatment extent of the retinal detachment. Our retinoblastoma practice has established the safety and accuracy of this external beam radiotherapy technique, which was adapted for use under local anaesthesia to the eye in the two cases reported here. Having established the safety and efficacy of this treatment, we would now recommend the earlier treatment of patients such as these in the expectation that visual deterioration would be arrested at an earlier stage.

In conclusion, radiotherapy remains an important form of treatment for orbital angiomas, and with the lesser morbidity of modern methods earlier treatment than previously may be indicated. Although radiotherapy for any benign lesion requires careful individual consideration, this treatment method remains appropriate, important, and effective therapy for some patients.

It is with great pleasure that we thank Mr J Hungerford and Mr D Taylor for referring patients and Miss $T$ Cocks for typing the manuscript.

\section{References}

1 Walter J. On the treatment of cavernous haemangioma with

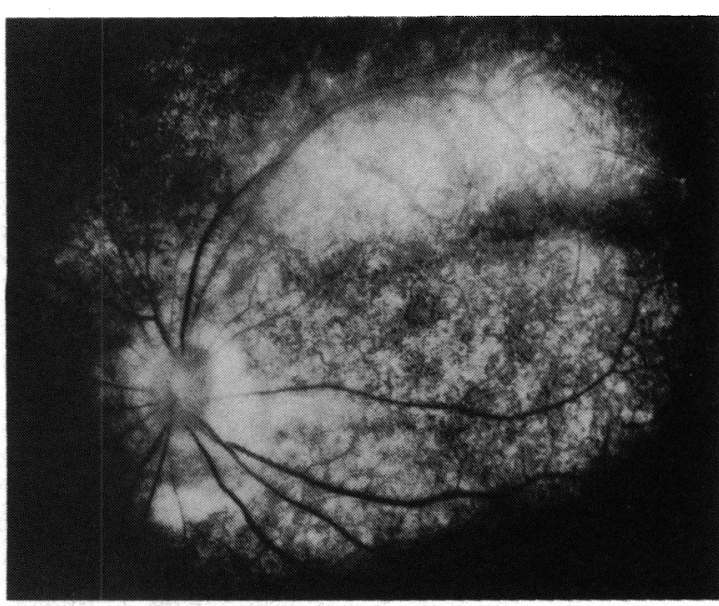

Fig. 2B Taken six months after radiotherapy. There is more rapid clearing of the dye; the retina has flattened and become reattached.

special reference to spontaneous regression. J Fac Radiol 1953; 5: 134-40.

2 Bowers RE, Graham EA, Tomlinson KM. The natural history of the strawberry nevus. Arch Dermatol 1960; 82: 667-80.

3 Martin LW, MacCollum DW. Hemangiomas in infants and children. Am J Surg 1961; 101: 571-80.

4 Zarem HA, Edgerton MT. Induced resolution of cavernous hemangiomas following prednisolone therapy. Plast Reconstr Surg 1967; 39: 76-83.

5 de Venecia G, Lobeck CC. Successful treatment of eyelid hemangioma with prednisone. Arch Ophthalmol 1970; 84: 98-102.

6 Hiles DA, Pilchard WA. Corticosteroid control of neonatal hemangiomas of the orbit and ocular adnexa. Am J Ophthalmol 1971; 71: 1003-8.

7 Kushner BJ. Local steroid therapy in adnexal hemangioma. Ann Ophthalmol 1979; 11: 1005-9.

8 Zak TA, Morin JD. Early local steroid therapy of infantile eyelid hemangiomas. J Pediatr Ophthalmol Strabismus 1981; 18: 25-7.

9 Henderson JS. Orbital tumours. Philadelphia: Saunders, 1973: 124-67.

10 Haik BG, Jakobiec FA, Ellsworth RM, Jones IS. Capillary hemangioma of the lids and orbit: an analysis of the clinical features and therapeutic results in 101 cases. Trans Am Acad Ophthalmol 1979; 86: 760-89.

11 Bek V, Zahn K. Cataract as a late sequel of contact roentgen therapy of angiomas in children. Acta Radiol (Stockh) 1960; 54: 443-8.

12 Harnett AN, Hungerford J, Lambert GD, et al. Improved external beam radiotherapy for the treatment of retinoblastoma. Br J Radiol 1987; 60: 753-60.

Accepted for publication 25 March 1987. 